\title{
CAMBRIDGE Mathematics
}

\section{Now in paperback}

\section{A Guide to Quantum Groups}

\section{CHARI and A. N. PRESSLEY}

A comprehensive view of quantum groups and their applications. The authors build on a self-contained account of the foundations of the subject and go on to treat the more advanced aspects concisely and with detailed references to the literature. Thus this book can serve both as an introduction for the newcomer, and as a guide for the more experienced reader. All who have an interest in the subject will welcome this unique treatment of quantum groups.

f22.95 net PB $0521558840 \quad 672$ pp. 1995

\section{Multivalent Functions}

\section{Second Edition}

\section{W. K. HAYMAN}

The second edition of this celebrated book is the first to contain a full and self-contained proof of De Branges' theorem. Every chapter has been updated and many of the original proofs have been simplified. It will be essential for all interested in complex function theory.

E35.00 net HB $0521460263 \quad 275$ pp. 1994

Cambridge Tracts in Mathematics 110

\section{Skew Fields}

Theory of General Division Rings

\section{P. M. COHN}

Based on the author's LMS lecture note volume Skew Field Constructions, the present work offers a comprehensive account of skew fields. Numerous exercises test the reader's understanding, presenting further aspects and open problems in a concise form. The notes and comments at the end of each chapter provide an historical background.

E55.00 net HB $0521432170 \quad 516 \mathrm{pp} . \quad 1995$

Encyclopedia of Mathematics and its Applications 57

\section{Introduction to Analytic and Probabilistic Number Theory}

\section{G. TENENBAUM}

Translated by C. B. Thomas

A self-contained introduction to analytic methods in number theory. It offers to students and beginning researchers a systematic and consistent account of the subject but will also be a convenient resource and reference for more experienced mathematicians.

f45.00 net HB $0521412617 \quad 464$ pp. 1995

Cambridge Studies in Advanced Mathematics 46

\section{Clifford Algebras and the Classical Groups}

\section{PORTEOUS}

Here, Ian Porteous has reworked his previous book on this subject, Topological Geometry, and has expanded and added material to bring the theory of Clifford algebras to the fore. This treatment of the theory of Clifford algebras will be welcomed for its clarity and detail.

f30.00 net HB $0521551773 \quad 304$ pp. 1995

Cambridge Studies in Advanced Mathematics 50

Cambridge books are available from good bookshops, alternatively phone UK + $44(0) 1223325970$ to order direct using your credit card, or fax UK $+44(0) 1223315052$. For further information on any of our mathematics titles, please email Giulia Williams on science@cup.cam.ac.uk or browse our Worldwide Web server http://www.cup.cam.ac.uk

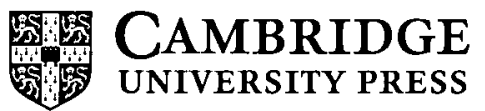




\title{
Mathematical Structures in Computer Science
}

\author{
Editor-in-Chief \\ G. Longo, Laboratoire d'Informatique (CNRS) et DMI, Ecole Normale Superieure, 45 rue d'Ulm, 75005, Paris \\ (email: longo@dmi.ens.fr)
}

Associate Editors

P. L. Curien, LIENS, 45 rued'Ulm, 75230 Paris (email: curien@dmi.ens.fr)

A. M. Pitts, Computer Laboratory, University of Cambridye, Pembroke St, Cambridge CB2 3QG

\begin{abstract}
Mathematical Structures in Computer Science (MSCS) is a new journal of excellence in theoretical computer science which focuses on the application of ideas from the structural side of mathematics and mathematical logic to computer science. The journal bridges the gap berween theoretical contributions and software design, publishing original papers or broad surveys with original perspectives in all areas of computing, provided that ideas or results from algebra, geometry or category theory form a basis for the work.

MSCS is distinct from existing titles in that it specialises in the art of applying mathematics of genuine interest and general applicability to computer science; its objective is to promote the useful application of high level mathematics to language design and software implementation. The journal increases the circulation of new results in this fast growing area.
\end{abstract}

\section{Essential reading for:}

- Mathematicians with interests in computer science

- Theoretical computer scientists

- Computer scientists working in language development or formal methods

\section{Recent articles}

The glueing construction and lax limits Harold Simmons

Dynamic labeled 2-structures

A. Ehrenfeucht and $G$. Rozenberg

Interaction Systems I: The theory of optimal reductions Andrea Asperti and Cosimo Laneve

Languages under concatenation and shuffling Steven T. Tschantz

Connected limits, familial representability and Artin glueing Aurelio Carboni and Peter Johnstone

Sound and complete axiomatisations of call-by-value control operators

Martin Hofmann

Reductions in Intuitionistic Linear Logic

Sergei Soloviev

Fibrations with indeterminates: contextual and functional completeness for polymorphic lambda calculi Claudio Hermida and Bart Jacobs

\section{Subscription information}

Mathematical Structures in Computer Science, Volume 6, 1996: February, April, June, August, October and December 1996: 1 144. Delivery by airmail $\{22.00$ per year extra. ISSN 0960-1295

\section{Take a closer look - FREE!}

I $\square$ Please send me a free sample copy of:

| Mathematical Structures in Computer Science

$\square$ Please send me further information

I

Name

| Address

1

1

Send to: Journals Marketing Department,

Cambridge University Press, FREEPOST*, The

Edinburgh Building, Cambridge, Cв2 IBR, UK.

Tel: $+44(0) 1223325806$ Fax: $+44(0) 1223315052$

Email: journals_marketing@cup.cam.ac.uk

('no postage stamp necessary if posted in UK)

In USA, Canada \& Mexico, write to: Cambridge University Press, 40 West 20th Street, New York, NY 10011-4211, USA.

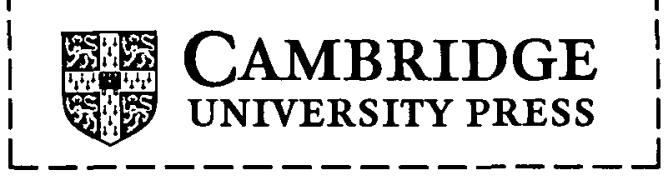




\section{Instructions for Authors}

Editorial policy The journal welcomes submissions in any of the areas of Combinatorics, Probability, or Computer Science, not just those dealing explicitly with relations between the three. Its scope covers combinatorics in a broad sense, including classical and algebraic graph theory, probabilistic methods, random structures, combinatorial probability and limit theorems for random combinatorial structures; and the theory of algorithms, including complexity theory, randomised algorithms, probabilistic analysis of algorithms, computational learning theory and optimisation.

Submission of manuscripts Papers may be submitted to any member of the Editorial Board. Three copies should be sent accompanied by the author's address, telephone and fax number, and if possible, an electronic mailing address.

Submission of a paper is taken to imply that it has not been previously published and that it is not being considered for publication elsewhere. Upon acceptance of a paper, the author will be asked to transfer copyright to the publisher.

Authors are encouraged to submit papers electronically by sending a LaTex file to cpc@dpmms.cam.ac.uk; this file should include the figures (line figures only) and all authordefined macros. Authors using LaTex should use the CPC LaTex style file which can be obtained using anonymous FTP from the internet address ftp.cup.cam.ac.uk. Go into the directory /pub/texarchive/journals/latex/cpc where you will find a concatenated file called cpc.all. The file cpc.all contains readme.txt, cpc.sty and cpcguide.tex. If you Tex cpcguide.tex you will get a full set of instructions for using the style file. In case of difficulties obtaining these files, there is a help-line available via e-mail; please contact texline@cup.cam.ac.uk. While use of CPC LateX style file is preferred, ordinary LaTeX or plain Tex files can also be accepted.

On final acceptance of a paper, authors should send the LaTeX source code on disc to the editorial office, together with a hard copy produced using the same file. Discs should be in Apple Mac or PC format and will not be returned. The publisher reserves the right to typeset any article by conventional means if the author's TeX code presents problems in production.

Layout of conventional manuscripts Papers should be typewritten in double spacing throughout, on one side of the paper. Please avoid footnotes if possible. Papers should begin with an abstract of not more than $\mathbf{3 0 0}$ words and should end with a brief concluding section.

Illustrations Figures should be drawn in indian ink on good quality white paper or produced by computer to comparable quality. Wherever possible they will be reproduced with the author's original lettering. Originals of figures should not be sent until the paper has been accepted. A list of captions should be attached separately.

References References should be listed in alphabetical order at the end of the main text. Please include the article title in the reference, which should be in the order: author's surname, initials; year in parentheses; article title; journal name abbreviated in accordance with the World List of Scientific Periodicals (4th edn); volume number; inclusive page numbers. For books and conference proceedings, place of publication and publisher (and Editor(s) if appropriate) should be included. In the text, references should be cited as [1].

Proof reading Typographical or factual errors only may be changed at proof stage. The publisher reserves the right to charge authors for correction of non-typographical errors. No page charge is made.

Offprints 50 offprints of each article will be supplied free to each first named author. Extra offprints may be purchased from the publisher if ordered at proof stage. 


\section{Combinatorics, Probability \& Computing}

\section{CONTENTS}

Perfect Matchings in Random r-regular, s-uniform Hypergraphs COLIN COOPER, ALAN FRIEZE, MICHAEL MOLLOY AND BRUCE REED

The Harmonious Chromatic Number of Bounded Degree Trees KEITHEDWARDS

An Upper Bound on Zarankiewicz' Problem ZOLTÁN FÜREDI

Precoloring Extension III: Classes of Perfect Graphs

M. HUJTER AND ZS. TUZA

Excluding Minors in Cubic Graphs

K. KILAKOS AND B. SHEPHERD

Topological cliques in graphs II

JÁNOS KOMLÓS AND ENDRE SZEMERÉDI

Short Proof of Galvin's Theorem on the List-chromatic Index of a Bipartite Multigraph

TOMAŽ SLIVNIK

Printed in Great Britain by the University Press, Cambridge

\section{CAMBRIDGE UNIVERSITY PRESS}

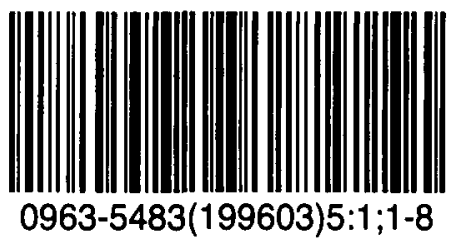

